\title{
Highly efficient all-optical switching of magnetization in GdFeCo microstructures by interference-enhanced absorption of light
}

\author{
M. Savoini, ${ }^{1,}{ }^{*}$ R. Medapalli, ${ }^{1}$ B. Koene, ${ }^{1}$ A. R. Khorsand,${ }^{1}$ L. Le Guyader,,${ }^{2, \dagger}$ L. Duòo, ${ }^{3}$ M. Finazzi, ${ }^{3}$ A. Tsukamoto,,${ }^{4,5}$ A. Itoh, ${ }^{4}$ \\ F. Nolting, ${ }^{2}$ A. Kirilyuk, ${ }^{1}$ A. V. Kimel, ${ }^{1}$ and Th. Rasing ${ }^{1}$ \\ ${ }^{1}$ Radboud University Nijmegen, Institute for Molecules and Materials, Heyendaalseweg 135, Nijmegen, The Netherlands \\ ${ }^{2}$ Paul Scherrer Institut, CH-5232 PSI-Villigen, Switzerland \\ ${ }^{3}$ Dipartimento di Fisica, Politecnico di Milano, Piazza Leonardo da Vinci 32, I-20133 Milano, Italy \\ ${ }^{4}$ College of Science and Technology, Nihon University, 7-24-1 Funabashi, Chiba, Japan \\ ${ }^{5}$ PRESTO, Japan Science and Technology Agency, 4-1-8 Honcho Kawaguchi, Saitama, Japan
}

(Received 19 July 2012; published 18 October 2012)

\begin{abstract}
Employing magnetization-sensitive microscopy techniques, we address the light-induced magnetization dynamics of patterned samples of rare-earth transition metal alloys. We experimentally find that smaller structures require a lower energy density to undergo all-optical switching of the magnetization. With the aid of simulations we explain this reduction in terms of enhanced light absorption by interference of the light within the structure. This results in a decrease of about $60 \%$ in the energy densities required for all-optical switching compared with those in continuous thin films of the same alloy. Moreover, we envisage that an energy lower than $10 \mathrm{fJ}$ should be sufficient to switch a $20 \times 20 \mathrm{~nm}^{2}$ structure.
\end{abstract}

DOI: 10.1103/PhysRevB.86.140404

PACS number(s): 75.40.Gb, 75.60.Jk, 85.70.Ay

\section{INTRODUCTION}

The incessant demand for higher and higher recording densities in magnetic hard drives requires continuous efforts for further magnetic bit miniaturization. Several efficient techniques have already been developed for recording and retrieving nanosize magnetic bits, but the rate of writing and reading single data is still limited to several ns. ${ }^{1}$ One way for obtaining a faster working speed together with energyefficient data storage is the use of all-optical switching (AOS) in rare-earth transition metal ferrimagnetic thin films, ${ }^{2}$ in which unsurpassed recording rates have been demonstrated. ${ }^{3,4}$ The switching process here is ignited by the absorption of photons ${ }^{5,6}$ and requires an amount of energy which compares favorably with present-day magnetic recording technologies. ${ }^{7,8}$

By carefully tuning the laser fluence and exploiting the threshold barrier that is characteristic for the optically induced reversal, ${ }^{2,8}$ it is possible to confine the AOS within a few tens of squared micrometers. Such a dimension is orders of magnitude larger than the current bit size in magnetic data storage. This problem can be circumvented by patterning the magnetic films and realizing micro- and nanosized structures. ${ }^{9,10}$ However, a thorough understanding of how structuring down to dimensions comparable to or smaller than the optical wavelength affects AOS key parameters, such as switching speed, and fluence threshold, is still lacking.

In this Rapid Communication, we present our results on ultrafast magnetization dynamics and reversal in different microstructures realized in a GdFeCo thin film. We show that there are strong effects of light interference within these structures, leading to an enhanced energy absorption for smaller areas. This makes AOS an ideal candidate for extremely energy-effective and fast magnetic recording.

\section{SAMPLES AND EXPERIMENTAL SETUP}

The sample consists of a series of patterned squared areas with different dimensions, fabricated via liftoff techniques in a film of $\mathrm{Gd}_{26} \mathrm{Fe}_{64.5} \mathrm{Co}_{9.5}$, as reported in Ref. 9. The structure and thickness (number between parentheses in $\mathrm{nm}$ ) of the layers is as follows: glass/ $\mathrm{Ti}(2) / \mathrm{Pt}(8) / \mathrm{SiN}(5) / \mathrm{GdFeCo}(20) / \mathrm{SiN}(20)$.

To address the magnetization dynamics in a single structure down to $1 \times 1 \mu \mathrm{m}^{2}$, we modified a commercial confocal microscope ( $\alpha$-SNOM, WiTec GmbH, Germany) to obtain femtosecond temporal and submicrometer spatial resolution. ${ }^{11}$ This setup gives the possibility to work in ambient conditions and with a table-top setup; moreover, it offers several advantages in terms of simplicity and temporal resolution compared with other techniques (see, for example, Refs. 12-14).

We employ an amplified Ti:sapphire system (repetition rate $f=250 \mathrm{kHz}$, output power $P_{\mathrm{av}}=1.5 \mathrm{~W}$, emission wavelength $\lambda=800 \mathrm{~nm}$, pulse duration $\tau=60 \mathrm{fs}$ ). We split the laser beam into two parts with a $90 \%-10 \%$ beam splitter to set up a collinear pump-probe scheme. The pump beam is doubled in frequency with a nonlinear crystal. Both beams, after being delayed with respect to each other, are linearly polarized and enter into the confocal microscope. Light is focused on the sample by an Olympus $40 \times(\mathrm{NA}=0.55)$ microscope objective. Due to chromatic aberrations, the focal planes differ for pump $(\lambda=400 \mathrm{~nm})$ and probe $(\lambda=800 \mathrm{~nm})$. We place the sample in the probe focal plane, where we have a diffraction-limited probe spot of $\sigma_{\text {probe }}=800 \mathrm{~nm}$ while the larger pump spot size is $\sigma_{\text {pump }}=5 \mu \mathrm{m}$ full width at half maximum (FWHM). The dimensions of the beams have been carefully evaluated using Liu's method. ${ }^{15}$

After the sample, the beams are collected by a Nikon objective $(100 \times, \mathrm{NA}=0.8)$, pass the analyzer, and are focused onto the detector. We exclude contributions from the pump beam with a long-pass filter (RG695). The temporal resolution has been measured to be $300 \mathrm{fs}$ with an autocorrelation technique after both objectives. We thus estimate the pulse duration in the sample plane to be lower than $200 \mathrm{fs}$.

Figure 1 shows the static characterization of the magnetization in different structures, performed with the probe beam $\left(\sigma_{\text {probe }}=800 \mathrm{~nm}\right)$. The fact that the probe beam is smaller than the structure size allows for a direct comparison of the 


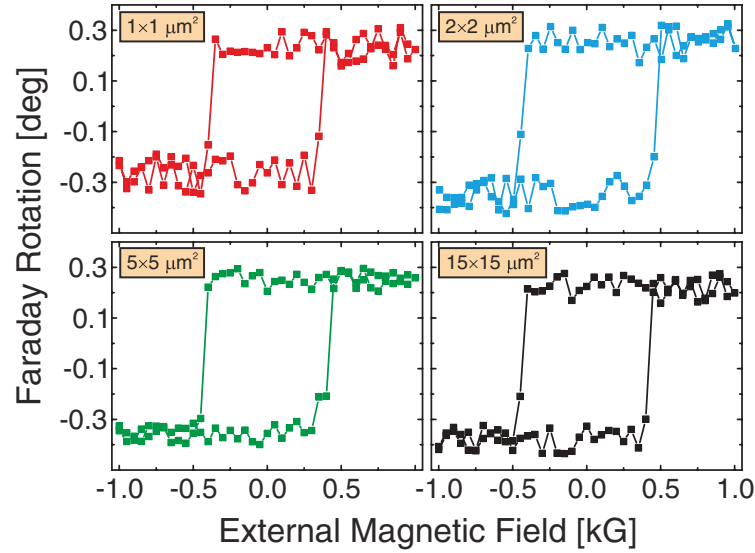

FIG. 1. (Color online) Hysteresis cycles for structures with different lateral sizes, showing that the magnetic properties of each studied area do not significantly differ from each other.

measured hysteresis loops. The samples show out-of-plane anisotropy, a high Faraday rotation (about $0.6^{\circ}$ at $\lambda=800 \mathrm{~nm}$ ), and a coercive field of about $H_{c}=400 \mathrm{G}$. It is important that structures with different lateral sizes present similar magnetic properties so that we can directly compare their magneto-optical properties.

\section{EXPERIMENTAL RESULTS AND DISCUSSION}

With the aforedescribed optical microscope we performed pump-probe measurements, exciting the sample magnetization with an intense pump pulse and recording the Faraday rotation of the probe as a function of the relative delay between pump and probe. The sample magnetization is always saturated along the easy axis with the use of a permanent magnet in close proximity to the sample $(\approx 800 \mathrm{G}$ at the position of the sample), such that stroboscopic measurements can be performed.

Figure 2(a) presents an example of the pump-probe magnetization dynamics obtained as a function of the fluence for the $15 \times 15 \mu \mathrm{m}^{2}$ structure. By increasing the laser fluence we see an increasing demagnetization. Note that for fluences below $3 \mathrm{~mJ} / \mathrm{cm}^{2}$ the magnetization relaxes back to the initial state with an exponential behavior with a characteristic time of 100-150 ps. This relaxation becomes slower for demagnetizations higher than $70 \%$ (or alternatively laser fluences higher than $3 \mathrm{~mJ} / \mathrm{cm}^{2}$ ), at the point that it cannot be fitted by a single exponential but shows a piecewise linear trend. We can explain this trend as a loss of correlation in the local exchange interaction due to the high electronic temperature reached by the system, making the relaxation dynamics slower than exponential. This trend is in qualitative agreement with calculations based on Landau-Lifshitz-Bloch equations ${ }^{16}$ and atomistic simulations. ${ }^{17}$

In the case shown in Fig. 2(a), for fluences above $5.5 \pm$ $0.55 \mathrm{~mJ} / \mathrm{cm}^{2}$, magnetization switching is induced. This observation is in agreement with recent experiments which show that AOS is subjected to a threshold fluence, ${ }^{8}$ and has been ascribed to ultrafast nonequilibrium dynamics of the $\mathrm{Gd}$ and $\mathrm{FeCo}$ sublattices. ${ }^{5,6}$ The reversal occurs within $50-100$ ps, similar to previously reported optical measurements in continuous films. ${ }^{18}$ In the absence of any external magnetic field, this

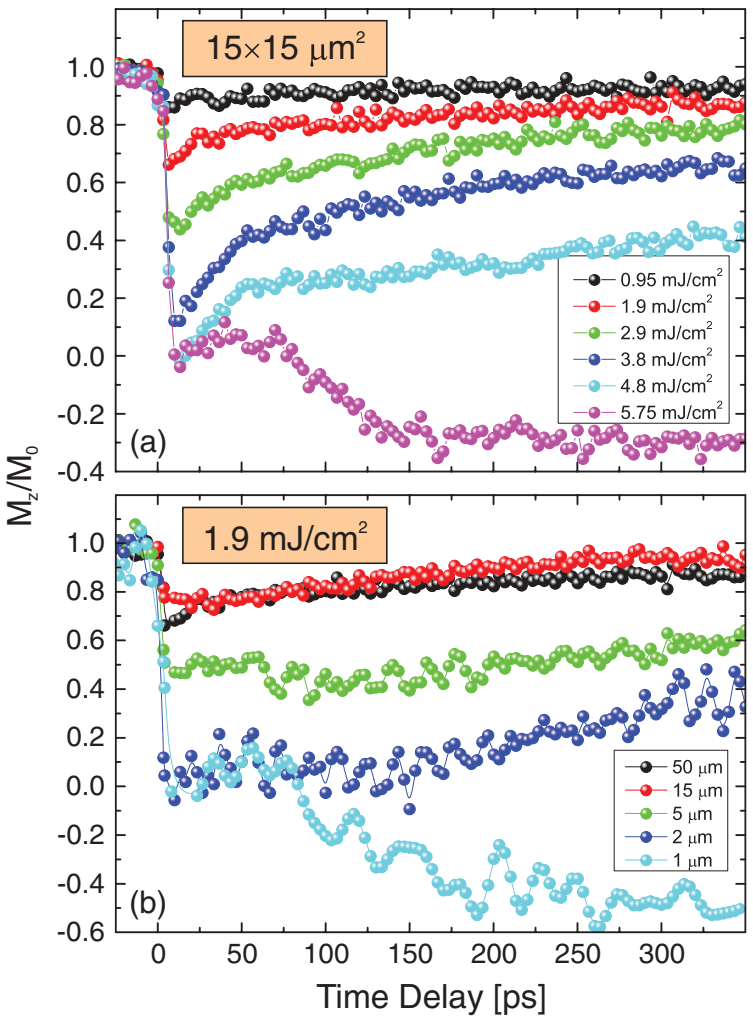

FIG. 2. (Color online) (a) Magnetization dynamics as a function of the fluence for the structure having $15 \times 15 \mu \mathrm{m}^{2}$ area. It is visible that at a fluence of about $5.5 \mathrm{~mJ} / \mathrm{cm}^{2}$ we induce a switching event, which then starts to slowly relax back due to the presence of the external restoring magnetic field. (b) Magnetization dynamics at a fixed fluence of $1.9 \pm 0.2 \mathrm{~mJ} / \mathrm{cm}^{2}$ for different structure dimensions. A strong dependence is visible as we measure a higher demagnetization upon decreasing the structure dimensions. For the smallest structure AOS is induced.

negative relaxation would continue to reach the full switching (level $M_{z} / M_{0}=-1$ ). In the case of our measurements, we access only the first $500 \mathrm{ps}$, i.e., when the temperature of the illuminated spot is still elevated and the external field is acting against the switched domain. These factors act together to saturate the probed magnetization switching at the level $M_{z} / M_{0}=-0.3$.

A similar trend is observed if we study the pump-probe dynamics for a fixed pump fluence, for example, $1.9 \pm$ $0.2 \mathrm{~mJ} / \mathrm{cm}^{2}$ (twice as low as the one required for AOS in a continuous film), upon reducing the structure dimensions, as presented in Fig. 2(b). For structures with dimensions of $50 \times 50 \mu \mathrm{m}^{2}$ and $15 \times 15 \mu \mathrm{m}^{2}$, both much larger than the pump and probe beam sizes, the effect on the magnetization is practically the same. However, the initial demagnetization considerably increases upon further reducing the structure dimension. Surprisingly, we measured $50 \%$ and basically full demagnetization in the $5 \times 5 \mu \mathrm{m}^{2}$ and $2 \times 2 \mu \mathrm{m}^{2}$ structures, respectively. Eventually even all-optical switching is observed in $1 \times 1 \mu \mathrm{m}^{2}$ structures.

Notably, the different dimensions do not directly affect the initial demagnetization speed which occurs on average within 1 ps. Naively one would, moreover, expect that the pumpinduced changes should be triggered by a constant energy 




FIG. 3. (Color online) Energy requirements for 100\% demagnetization, all-optical switching, and the damage threshold as a function of the structure size. Inset: Experimental conditions reproducing in scale the dimensions of pump and probe beams.

density, thus we expect no dependence as a function of the dimension.

The experimental conditions are sketched in the inset of Fig. 3, where the pump lateral size is about five times larger than the probe one. Thus the probed area is excited by a homogeneous (practically constant) laser fluence. Figure 3 presents, respectively, the trend for the energy densities required to induce $100 \%$ demagnetization, AOS, and sample damage, i.e., an irreversible change of the magnetization status of the illuminated area, as a function of the sample size. Indeed, for the larger structures $\left(15 \times 15 \mu \mathrm{m}^{2}\right.$ and $50 \times 50 \mu \mathrm{m}^{2}$ ) we do not measure any dependence in the energy requirements as a function of the dimension. Conversely, a clear deviation is present for the smaller ones, and a strong reduction of the required energy densities is apparent. Thus we have experimentally demonstrated that optically induced effects, as AOS, become energetically more favorable reducing the structure size. For example, pattern sizes of $1 \times 1 \mu \mathrm{m}^{2}$ require three times lower energy compared with larger ones (or continuous films). This finding makes AOS one of the most energy-effective means to control the magnetization of a sample. Therefore understanding the responsible mechanisms that are making the energy absorption more effective in smaller areas is a key point both for fundamental research and possible applications.

Since demagnetization and AOS are events induced by a single optical pulse and occur on a picosecond time scale, we can exclude that the reduced heat capacity and/or the limited electron diffusion of a smaller structure may play an important role. In fact, heat diffusion and hot electron transport and thermalization occur within longer time scales for spatial distances of the order of $1 \mu \mathrm{m}$ or larger [about several tens of ns (Ref. 19)]. Thus no influence on the trends reported in Fig. 3 is expected. On the other hand, the drop of the required energy densities can be explained by an increased absorption due to interference effects of the light when its wavelength and the focused dimension are of the same order as the sample size.
We address this possibility through simulations, as presented in the following section.

\section{FINITE DIFFERENCE TIME DOMAIN MODELING}

Finite difference time domain (FDTD) simulations have already proved to be an effective tool to understand interference effects, ${ }^{20}$ and thus we have performed FDTD calculations ${ }^{21}$ to investigate the lateral confinement and interference of the optical field distribution within the magnetic layer.

We have simulated, with a $5 \times 5 \times 1 \mathrm{~nm}^{3}$ mesh, the multilayer structures, using refractive indices extracted from ellipsometric measurements. ${ }^{8}$ The dimension and focusing of the pump beam are chosen to match the experimental values. The simulations were performed from a structure size of $20 \times 20 \mathrm{~nm}^{2}$, basically equivalent to the actual bit size in present-day hard drives, up to $15 \times 15 \mu \mathrm{m}^{2}$.

Figure 4(a) shows the resulting intensity profile across a line through the center of the structures. It is apparent that reducing the structure dimension below the beam size ( $\approx 5 \mu \mathrm{m}$ FWHM) leads to the formation of a standing wave pattern due to the scattering of the beam by the structure edges. From these electric field distributions we can calculate the integral of the absorbed energy density. Figure 4(b) shows the trend of the simulated absorbed energy density in the structures (squares) compared with the integrated profile of a Gaussian beam within the same area (circles). Regarding the former, we have calculated the integral per unit area of the absorbed
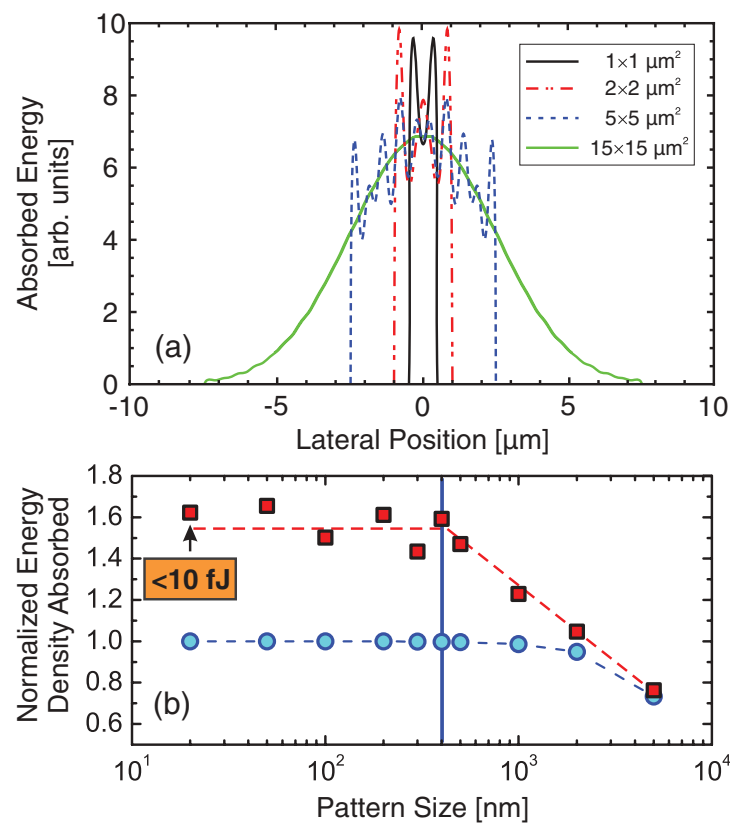

FIG. 4. (Color online) (a) Energy profiles of the electric field intensity taken at the center of the structures. The formation of a standing pattern due to the lateral confinement of the incoming radiation, when the dimensions of the structures are equal to or smaller than the beam size, is clearly visible. (b) Variation of the simulated energy distribution per unit area as a function of the structure lateral dimension, represented with a squared point, compared with the trend expected in the case of no interference effects present (circles). The expected energy needed to optically switch a $20 \times 20 \mathrm{~nm}^{2}$ domain is as low as $10 \mathrm{fJ}$. The vertical line indicates the pump beam wavelength. 
energy distribution within the patterned structure, while the latter is simply the integral per unit area of a Gaussian profile matching the absorbed profile of the $15 \times 15 \mu \mathrm{m}^{2}$ structure (where no standing pattern is present).

We find two separate behaviors: (i) Below $400 \times 400 \mathrm{~nm}^{2}$, no significant change in the absorption is visible. (ii) Above $400 \times 400 \mathrm{~nm}^{2}$ a steady decrease in the integrated absorbed energy density is visible with increasing pattern size. The difference between absorption with or without interference effects is remarkable. For structures smaller than $5 \times 5 \mu \mathrm{m}^{2}$, the integrated Gaussian distribution quickly reaches a saturation value. In contrast, the simulated energy distribution including interference continues to increase up to approximately the $400 \times 400 \mathrm{~nm}^{2}$ structure. Below this point it remains constant, but showing a value which is about $60 \%$ higher than the integrated Gaussian distribution. We would like to remind that $400 \mathrm{~nm}$ is also the wavelength of the pump beam. This is a clear indication that interference of light in (sub)micrometer structures plays a crucial role; in fact, we have an increase in absorption until the structure size becomes similar to the wavelength. Reducing the pattern size even further does not show any significant enhanced absorption.

Note that there is one difference between the experimental measurements of Fig. 3 and the simulated results presented in Fig. 4(b). In the former, a deviation from the expected constant energy density is visible already for the $5 \times 5 \mu \mathrm{m}^{2}$ structure, while in the latter a clear effect of the interference is present only for patterned areas smaller than $2 \times 2 \mu \mathrm{m}^{2}$. This difference originates from the different probed area in experiments and simulations. In the experimental results we are probing the magnetization dynamics locally, with a beam size of $800 \mathrm{~nm}$. In contrast, in the simulations we compute the absorbed energy integrated over the entire structure.

We have also evaluated the influence of the thickness of the structure in the interference effects. We simulate that these effects are stronger for thinner samples, while they tend to disappear when either the magnetic layer and/or the capping layers become thicker than $100 \mathrm{~nm}$.

As mentioned above, the lower heat capacity of smaller structures cannot explain the increased efficiency of AOS, while it may still play a role in the damaging of the structures, which occurs at longer time scales. Smaller structures, in fact, do not have lateral dissipation channels. Thus the temperature tends to increase more than in the larger ones, finally leading to irreversible changes.

The presented results have very interesting consequences for AOS at the nanoscale. For example, we can extrapolate the energy required to optically switch a nanosized domain. In patterned areas smaller than the pump dimension $\left(\sigma_{\text {pump }}=5 \mu \mathrm{m}\right)$ the integrated Gaussian profile corresponds to the experimentally measured peak fluence. Thus we are able to estimate the energy required for any structure size by using the following formula:

$$
E=F_{1} \frac{\alpha_{1}}{\alpha_{\max }} A,
$$

where $F_{1}$ is the switching threshold fluence and $\alpha_{1}$ the normalized absorption enhancement for the $1 \times 1 \mu \mathrm{m}^{2}$ structure plotted in Fig. 4(b), $\alpha_{\max }$ is the maximum absorption enhancement, and $A$ is the area of the patterned structure. Thus with a required switching fluence $F_{1}=2 \mathrm{~mJ} / \mathrm{cm}^{2}$, $\alpha_{1}=1.3, \alpha_{\max }=1.6$, and $A=4 \times 10^{-7} \mathrm{~cm}^{2}$, the estimated energy for a $20 \times 20 \mathrm{~nm}^{2}$ bit is $E<10 \mathrm{fJ}$. Such an energy would be a few orders of magnitude smaller than that used in current magnetoresistive random access memory (MRAM) technology, and even compares favorably with the low energy requirements for magnetic semiconductor switching. ${ }^{7,22}$

\section{CONCLUSIONS}

We have demonstrated that micropatterned samples show a strong decrease in the energy requirements for optically induced magnetization changes, such as all-optical switching. We explain this reduction by interference of light in (sub)micrometer sized structures, which leads to $60 \%$ higher light absorption than in continuous films. Finally, we envisage that by employing techniques such as near-field optics and especially plasmonic antennas to confine the impinging pulses, ${ }^{12,13,20}$ together with the scaling down to a $20 \times 20 \mathrm{~nm}^{2}$ structure size, it should be possible to optically switch structures employing the extremely low energy of $10 \mathrm{fJ}$. To ensure the thermal stability of such a small domain, other rare-earth transition metal compounds may be used, such as $\mathrm{TbFeCo}$, which presents more than one order of magnitude larger uniaxial anisotropy and thus thermal stability, while maintaining similar optical and magneto-optical properties.

\section{ACKNOWLEDGMENTS}

We thank A. van Roij and A. Toonen at Radboud University and A. Weber at PSI for technical support. This research has received funding from Stichting voor Fundamenteel Onderzoek der Materie (FOM), De Nederlandse Organisatie voor Wetenschappelijk Onderzoek (NWO), the European Union (EU) Nano Sci-European Research Associates (ERA) project FENOMENA, ERC Grant Agreement No. 257280 (Femtomagnetism), and EC FP7 [Grants No. NMP3-SL-2008214469 (UltraMagnetron), No. 214810 (FANTOMAS), and No. 281043 (FEMTOSPIN)].

\footnotetext{
*m.savoini@ science.ru.nl

†Present address: Helmholtz-Zentrum Berlin für Materialien und Energie GmbH, BESSY II, Albert-Einstein-Strasse 15, 12489 Berlin, Germany.

${ }^{1}$ A. Moser, K. Takano, D. T. Margulies, M. Albrecht, Y. Sonobe, Y. Ikeda, S. Sun, and E. E. Fullerton, J. Phys. D: Appl. Phys. 35, R157 (2002).
}

${ }^{2}$ C. D. Stanciu, F. Hansteen, A. V. Kimel, A. Kirilyuk, A. Tsukamoto, A. Itoh, and Th. Rasing, Phys. Rev. Lett. 99, 047601 (2007).

${ }^{3}$ K. Vahaplar, A. M. Kalashnikova, A. V. Kimel, D. Hinzke, U. Nowak, R. Chantrell, A. Tsukamoto, A. Itoh, A. Kirilyuk, and T. Rasing, Phys. Rev. Lett. 103, 117201 (2009).

${ }^{4}$ A. Kirilyuk, A. V. Kimel, and Th. Rasing, Rev. Mod. Phys. 82, 2731 (2010). 
${ }^{5}$ T. A. Ostler, J. Barker, R. F. L. Evans, R. W. Chantrell, U. Atxitia, O. Chubykalo-Fesenko, S. El Moussaoui, L. Le Guyader, E. Mengotti, L. J. Heyderman, F. Nolting, A. Tsukamoto, A. Itoh, D. Afanasiev, B. A. Ivanov, A. M. Kalashnikova, K. Vahaplar, J. Mentink, A. Kirilyuk, Th. Rasing, and A. V. Kimel, Nat. Commun. 3, 666 (2012).

${ }^{6}$ J. H. Mentink, J. Hellsvik, D. V. Afanasiev, B. A. Ivanov, A. Kirilyuk, A. V. Kimel, O. Eriksson, M. I. Katsnelson, and Th. Rasing, Phys. Rev. Lett. 108, 057202 (2012).

${ }^{7}$ J. Liu, E. Fullerton, O. Guteisch, and D. Sellmyer, Nanoscale Magnetic Materials and Applications (Springer, New York, 2009).

${ }^{8}$ A. R. Khorsand, M. Savoini, A. Kirilyuk, A. V. Kimel, A. Tsukamoto, A. Itoh, and T. Rasing, Phys. Rev. Lett. 108, 127205 (2012).

${ }^{9}$ L. Le Guyader, S. El Moussaoui, E. Mengotti, L. J. Heyderman, F. Nolting, A. Tsukamoto, A. Itoh, A. Kirilyuk, Th. Rasing, and A. V. Kimel, J. Magn. Soc. Jpn. 36, 21 (2012).

${ }^{10}$ L. Le Guyader, S. El Moussaoui, M. Buzzi, R. V. Chopdekar, L. J. Heyderman, A. Tsukamoto, A. Itoh, A. Kirilyuk, Th. Rasing, A. V. Kimel, and F. Nolting, Appl. Phys. Lett. 101, 022410 (2012).

${ }^{11}$ M. Savoini, F. Ciccacci, L. Duò, and M. Finazzi, Rev. Sci. Instrum. 82, 023709 (2011).
${ }^{12}$ M. Celebrano, P. Biagioni, M. Zavelani-Rossi, D. Polli, M. Labardi, M. Allegrini, M. Finazzi, L. Duò, and G. Cerullo, Rev. Sci. Instrum. 80, 033704 (2009).

${ }^{13}$ B. Nechay, U. Siegner, M. Achermann, H. Bielefeldt, and U. Keller, Rev. Sci. Instrum. 70, 2758 (1999).

${ }^{14}$ M. R. Freeman and B. C. Choi, Science 294, 1484 (2001).

${ }^{15}$ J. M. Liu, Opt. Lett. 7, 196 (1982).

${ }^{16}$ U. Atxitia, O. Chubykalo-Fesenko, N. Kazantseva, D. Hinzke, U. Nowak, and Chantrell, Appl. Phys. Lett. 91, 232507 (2007).

${ }^{17}$ N. Kazantseva, U. Nowak, R. W. Chantrell, J. Hohlfeld, and A. Rebei, Europhys. Lett. 81, 27004 (2008).

${ }^{18}$ C. D. Stanciu, A. Tsukamoto, A. V. Kimel, F. Hansteen, A. Kirilyuk, A. Itoh, and T. Rasing, Phys. Rev. Lett. 99, 217204 (2007).

${ }^{19}$ X. Xun, C. Peng, and M. Mansuripur, Appl. Opt. 41, 4596 (2002).

${ }^{20}$ B. Koene, M. Savoini, A. Kimel, A. Kirilyuk, and Th. Rasing, Appl. Phys. Lett. 101, 013115 (2012).

${ }^{21}$ Lumerical Solutions, www.lumerical.com.

${ }^{22}$ A. H. M. Reid, G. V. Astakhov, A. V. Kimel, G. M. Schott, W. Ossau, K. Brunner, A. Kirilyuk, L. W. Molenkamp, and Th. Rasing, Appl. Phys. Lett. 97, 232503 (2010). 